International Journal of Distributed and Parallel Systems (IJDPS) Vol.3, No.5, September 2012

\title{
RFID AND IP BASED OBJECT IDENTIFICATION IN UBIQUITOUS NETWORKING
}

\author{
Nisha Vaghela ${ }^{1}$ and Parikshit Mahalle ${ }^{2}$ \\ ${ }^{1}$ Department of Computer Engineering (Computer Network), SKN College of \\ Engineering, Pune University, Pune, INDIA \\ nisha_vaghela@rediffmail.com
}

${ }^{2}$ Department of Computer Engineering, SKN College of Engineering, Pune University, Pune, INDIA

parikshitmahalle@yahoo.com

\begin{abstract}
Ubiquitous networking is an integrated part of future networking technology that can provide capabilities for connecting all of objects (computers, human, PDAs, cell phones etc.) in future network. It has to meet the challenge of seamless connection for communication between human and objects in internet infrastructure. Unique object identification is very much important to make the communication between objects possible. RFID tag can be used as unique identifier to identify a physical object. Radio Frequency Identification (RFID) is a technology used for object identification of system. Internet Protocol (IP) address is used to provide logical identity to find the location of object for communication. In this paper, IP based RFID architecture for unique identification and tracking of object by considering mobility is proposed. RFID Agent (RA) is used to generate IP address based on RFID tags. In proposed solution, RFID-IP mapping is used to identify and track the location of object as RFID Tag ID can be used to generate unique identifier and IP can be used to find the location. RFID deployment is cost effective and $I P$ is being used as current internet structure. In this way RFID-IP mapping provides better solution for object identification in ubiquitous networking environment.
\end{abstract}

\section{KEYWORDS}

Internet of Things, Network \& Communication, RFID, Ubiquitous computing

\section{INTRODUCTION}

Ubiquitous computing is the method of enhancing computer use by making many computers available throughout the physical environment, but making them effectively invisible to the user [1]. The ubiquitous networking [2] capabilities support various classes of applications/services which require "Any Services, Any Time, Any Where and Any Devices" operation. This networking capability should support communication between human and objects. One objective of ubiquitous networking is to meet the challenge of seamless connection of anything (e.g., humans and objects) in internet infrastructure consisting of devices and network. In ubiquitous networking, huge amount of embedded and invisible devices as well as software components will be connected to the Internet, and these objects are expected to play an important role to provide convenience services to users. In such ubiquitous computing environment, users will utilize various applications through functional objects anytime and anywhere.

In the ubiquitous network environment, RFID is the wireless identification technique capable of reading without direct line of sight contact through a RFID reader. To enable ubiquitous computing applications, mechanism for internet is necessary. Generally, such kind of locating mechanism can be supported by unique identification system. Whenever a networked 
application would like to store data or change the configuration of the tags via remote readers, addressing of the tags via internet is required. The RFID tag ID cannot perform the IP networking by itself because of being only an object-person identifier. IP can be used to identify location of an object by providing logical address. Integration of RFID technology and IP networking is the key idea behind RFID-IP mapping. RFID-IP mapping can be used to identify the object uniquely and track the location of object also in case of object mobility. In this paper, IP based RFID management system using RFID agent is proposed. RFID agent is used to map RFID tag id that comes from RFID tag to unique IP address. Goal of this paper is to generate globally unique identification system for object through IP and RFID and to provide seamless mobility for a user using RFID tag.

The remainder of this paper is organized as follows: A brief review of related work is given in Section 2. Section 3 describes the system architecture design and implementation. Section 4 consists of experimentations and results. Section 5 presents the conclusion and future scope.

\section{RELATED WORK}

In 2009 [2], Identification technique to identify objects in ubiquitous networking to make object to object communication is proposed. In that solution, service ID's which includes identifiers like RFID, content ID, telephone number, URL/URI, etc and communication ID's like IPv4/IPv6 Address, MAC Address, etc... have been suggested to provide identification to objects.Service ID like RFID tag identifier can be used for physical identification of non-ip based object like patient or medicines in healthcare application.

EPCglobal [3] is the non-profit organization which is set up at October 2003 to standardize the automatic identification system technology based on radio frequency. Each Electronic Product Code (EPC) number is encoded with a header, identifying the particular EPC version used for coding the entire EPC number. An EPC manager number is defined, allowing individual companies or organizations to be uniquely identified; an Object class number identifies objects used within this organization, such as product types. Finally, a Serial number provides the unique identification of each individual object tagged by the organization. In 2002[4], EPC identification scheme with the IP address identification scheme was compared. As a conclusion it was derived that both identification schemes are very similar in structure, but neither of them can be used to replace the other scheme. Both of them are needed to do item-level identification and network communication because IP addresses cannot be used as unique item identifiers and EPCs cannot be used as routing addresses in their original intention.

In 2007 [5], use of objects EPCs to create their current IPv6 addresses was proposed. Replacement of the network prefix of an IPv6 address by the EPC was suggested, which only works for 64-bit EPCs. The reader accessing the tag generates this IP address from the EPC and transmits it to the corresponding EPCIS server. The reader stores the created IPv6 address as well. The EPCIS server holds information about the current IP address of particular tags. If someone wants to retrieve data from the tag, the EPCIS server is asked for the IPv6 address and the tag can be contacted.

The Dynamic Host Configuration Protocol (DHCP) [6] is a network configuration protocol for hosts on IP networks. Computers that are connected to IP networks must be configured before they can communicate with other hosts. The most essential information needed is an IP address, default route and routing prefix. DHCP eliminates the manual task by a network administrator. DHCP supports static, dynamic and manual allocation of IP addresses. In static allocation DHCP server statically binds IP address and physical address which remains persistent to client whereas in dynamic allocation, DHCP allocates IP address from the pool of IP addresses for constant time. If the lease time is finished, the client is assigned new IP address. In Manual 
allocation, host configuration is provided from DHCP server which can be used by the administrator to allocate IP address. DHCP server can dynamically assign IP address based on MAC address. As RFID tags are of various lengths, DHCP can't assign IP addresses until they are converted to 48 bits of MAC address.

In 2008[7], RFID networking mechanism using Address management agent is proposed. In this paper, idea of generating unique 48 bits virtual MAC address based on RFID tag was proposed which can be further used by the DHCP server to allocate IP address to RFID tag dynamically.

As DHCP server assigns IP address dynamically, after the lease time is finished, the client has to reclaim for new IP address which may not be same as given previously by DHCP on other network.

The related work done so far mainly focuses on EPC tags and tries to integrate IP technology into the EPCglobal network. In this paper we are trying to make use of all type of passive tags as modern tags do highly exceed the functionality of former EPC tags and has more capability to provide end-to-end communication. We are trying to merge the fruitful ideas given in related papers so that integration of RFID networking can happen over standard IP technology. In this way we are not limited to EPC technology but can handle also other passive RFID standards. Solution for object mobility to other network to provide seamless mobility is also proposed in this paper.

\section{System Architecture Design and implementation}

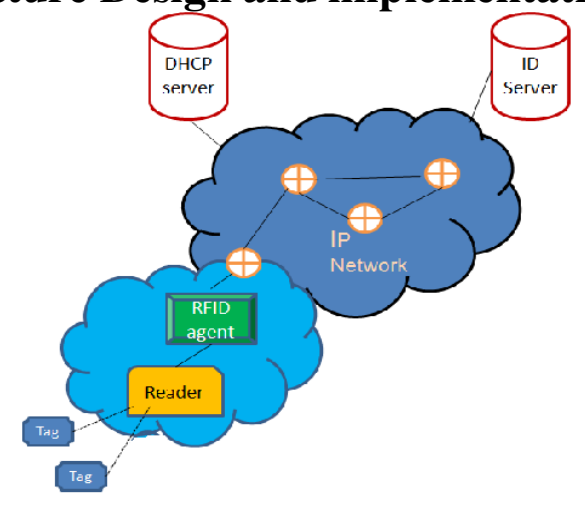

Figure 1. High level Architecture of RFID networking mechanism for object identification

High level architecture of proposed solution as shown in figure 1 consists of the main network, the DHCP server, the RFID agent (RA), the RFID reader, ID server, and the RFID Tag. Role of DHCP server is to get routing related information. The RFID reader is IP based reader that reads RFID tag or writes data in the RFID tag and works as IP router for tags. RFID readers are polling their environment continuously, so that they can sense each new tag in the field within an adequate time. RFID agent is an application attached to reader where the RFID tag ID is delivered. RFID agent performs mapping of tag identifier to IP address. It also registers the details associated with RFID tagged object in ID server. ID server plays dual role of storing RFID tag related information so that any Correspondent Node $(\mathrm{CN})$ can communicate to tag by taking information from ID server as well as it works like a home agent to provide seamless mobility in case if tag moves to another network. 


\subsection{Process for IP Address generation}

RFID tag identifiers are attached to every object. RFID tag ID is delivered to RFID reader. A delivered RFID tag ID is read by the RFID reader. The RFID reader delivers the read tag ID to the RFID agent (RA). RA transmits RFID tags information to DHCP server and also stores it in storage device. DHCP server gives routing related information from which net identifier part of IP address can be derived. RA generates unique host identifier of IP address from RFID tag identifier and merges both host identifier and net identifier to generate IP address for RFID tag. Generated RFID tag ID is stored in the storage device and registered to ID server. In the proposed network mechanism, the generated IP address is mapped with RFID tag ID. Procedure for object identification is explained using flow-chart in fig. 2 .

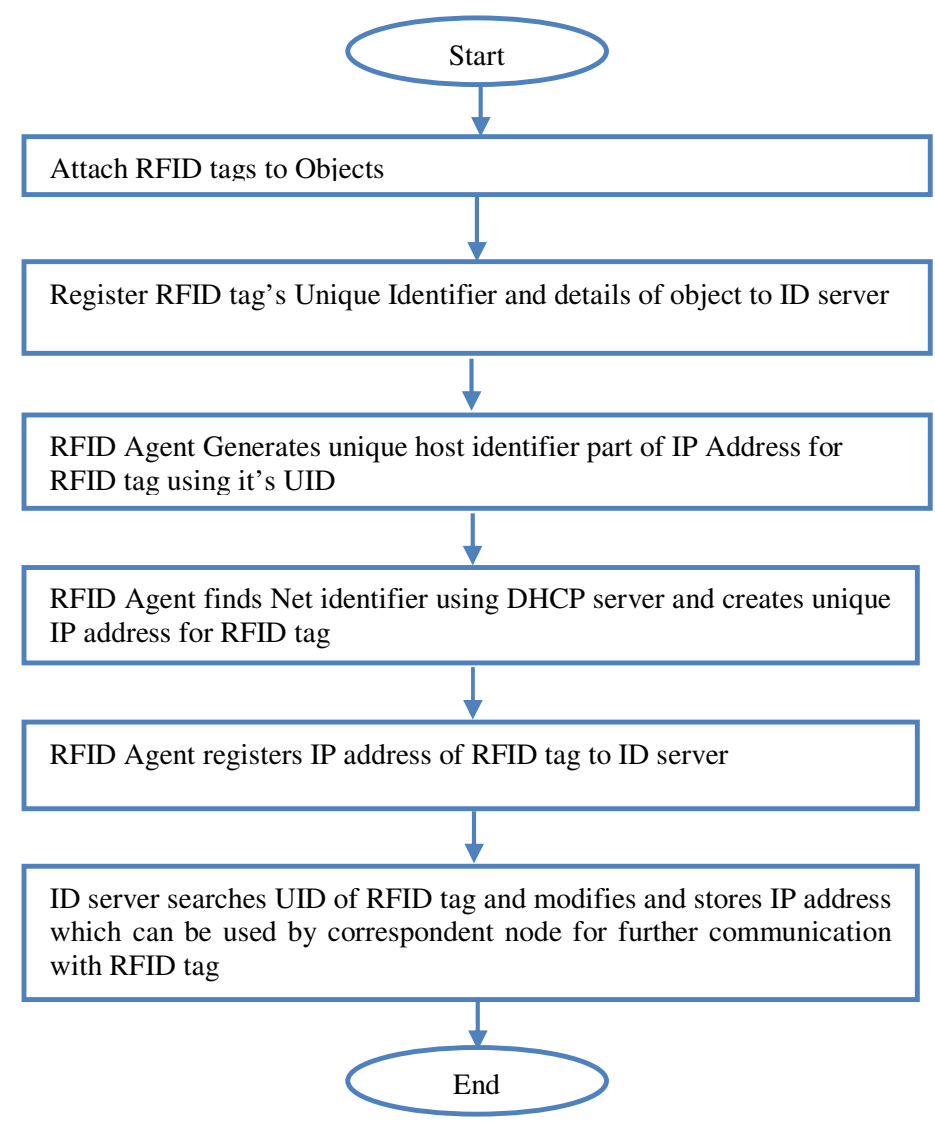

Figure 2: Flow chart for object identification 


\subsection{Object Mobility in proposed mechanism}

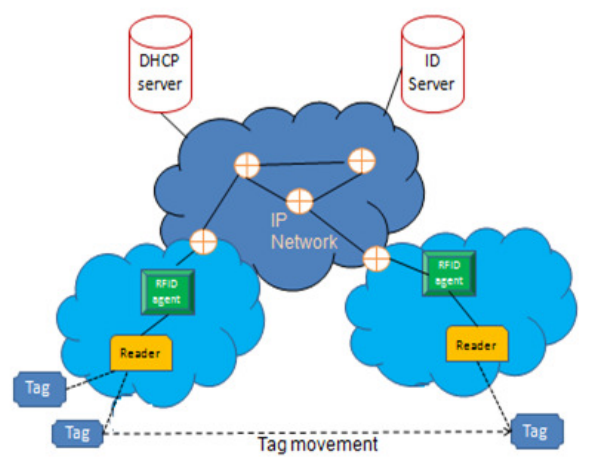

Figure 3: object identification considering mobility

In case of object mobility, RFID tags will be treated as mobile nodes. They use the RFID agent's (inside readers) as a "translators" to the IP network. There are some requirements that have to be fulfilled,

- According to mobile IP, there exists Home Agent (HA), which manages the tags and stores their current location. This HA will relay all IP packets for the corresponding tag.

- Each tag holds a unique IP address.

- The readers act as IP routers for the tags and manage the communication between tags and the $\mathrm{CN}$.

ID server can act as home agent for objects just like home agent in mobile IP. When a tag is newly issued, the ID server creates a new item in a database, where all assigned tags are registered with their UIDs and their Home Addresses. The Home Address consists of the net identifier of the ID server and the host identifier generated by RFID agent which uniquely identifies the tag at the Home Agent site. If a tag enters a reader field, a Care-Of Address (CoA) is created where the tag can currently be reached. It consists of the net identifier of the reader and the host identifier generated by RFID agent. RFID agent registers the RFID tag, its corresponding IP address (CoA) and agent\#1's IP address to ID server. ID server searches RFID tag ID by using key index and registers related information.

As shown in figure 3, when a user moves from an agent\#1(reader1) to an agent\#2(reader2), an agent\#2 is delivered the RFID tag ID and transmits it to DHCP server. The DHCP server gives net identifier part of the network to agent\#2. Agent\#2 generates unique host identifier part (same as that of agent\#1) based on RFID tag ID which is then combined to net identifier part and IP address is generated. An agent\#2 registers the RFID tag, agent\#2's IP address (same as reader2) and IP address of RFID tag (new CoA) to ID server. ID server searches the existing tag if any and modifies the details of RFID tag. 


\subsection{Communication procedure between corresponding node and mobile object}

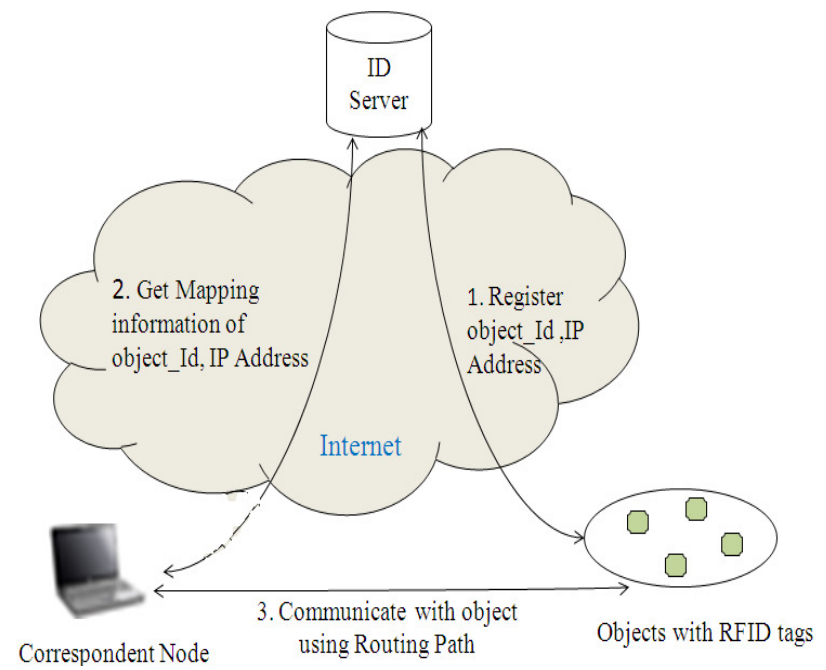

Figure 4: Communication between $\mathrm{CN}$ and object

As shown in figure 4, when Correspondent Node (CN) wants to communicate with RFID tag, it firstly contacts to ID server using home address of RFID tag. From the IP address, ID server can derive the CoA where the tag is present and query is forwarded to the destination IP address (CoA). The reader (agent) receives the query and searches for the mapped RFID tag ID by using the destination IP address forward the query to RFID tag. In this way communication between RFID tag and $\mathrm{CN}$ is established.

When RFID tag moves to agent\#2 during communication with $\mathrm{CN}$, it gets registered to ID server with CoA of agent\#2. All the data are buffered in ID server and then sent to agent\#2 using its CoA. Agent\#2 sends received data to RFID tag.

\section{EXPERIMENTAL SETUP AND RESULTS}

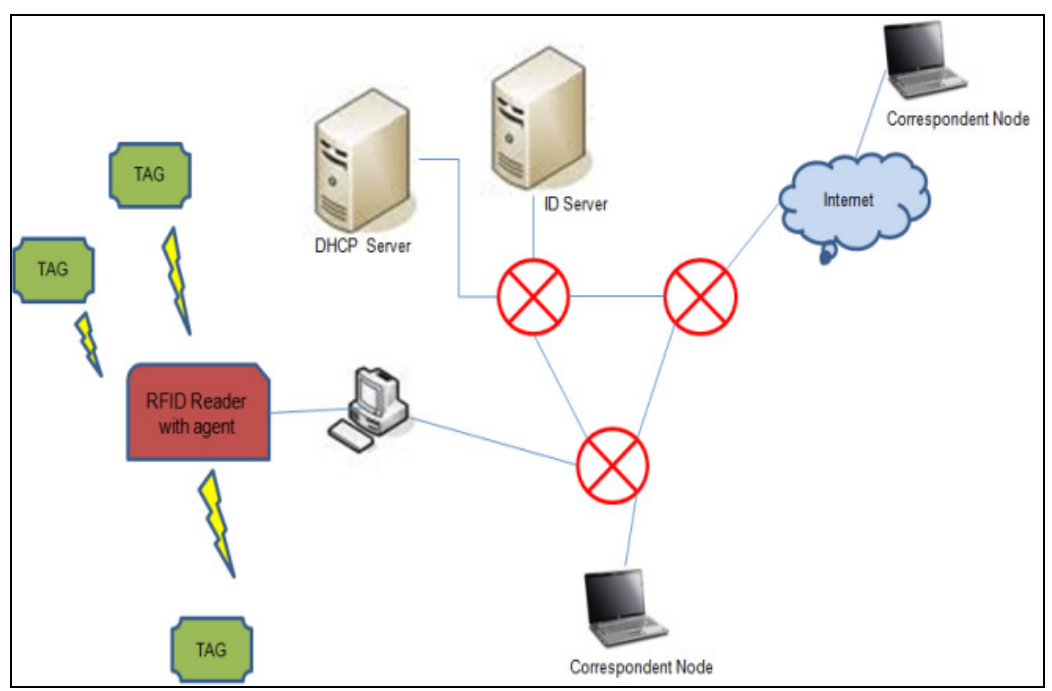

Figure 5: Experimental Setup 
Figure 5 shows Experimental setup for object identification and tracking for electronic health care system. As shown in figure 5, main network consists of RFID reader having RFID agent, RFID tagged patients, routers and DHCP server and ID server for health care.CN are doctors who want to monitor RFID tagged patient's health. Doctors may be within the network or in internet.

\section{Results}

RFID tag is read by reader and tag id is delivered to agent. Passive RFID tags are used as object identifiers. Here RFID agent is 8051 microcontroller based program which is a part of a reader. RA will convert tag id to IP address based on network prefix address of a network as net identifier and unique tag ID number as host identifier part. This IP address and details of RFID tag is then stored in ID server which is a database containing all the information of RFID tag. To store data in database we can use oracle server or Ms-Sql server. Once IP is generated by agent, both tag id and IP is given to ID server. ID server then stores IP of tag by mapping procedure of tag id. ID server also stores IP address of reader and agent (in this case both of them are having same IP as agent is part of reader) for respective tag id. Figure 6 and Figure 7 shows results.

Figure 6 shows RFID card allotment and IP registration to ID server when tag comes within the range of reader. Figure 7 shows report of readers where the tags are tracked with their corresponding IP address. Figure 8 shows result for tracking the objects if object is already registered to ID server.

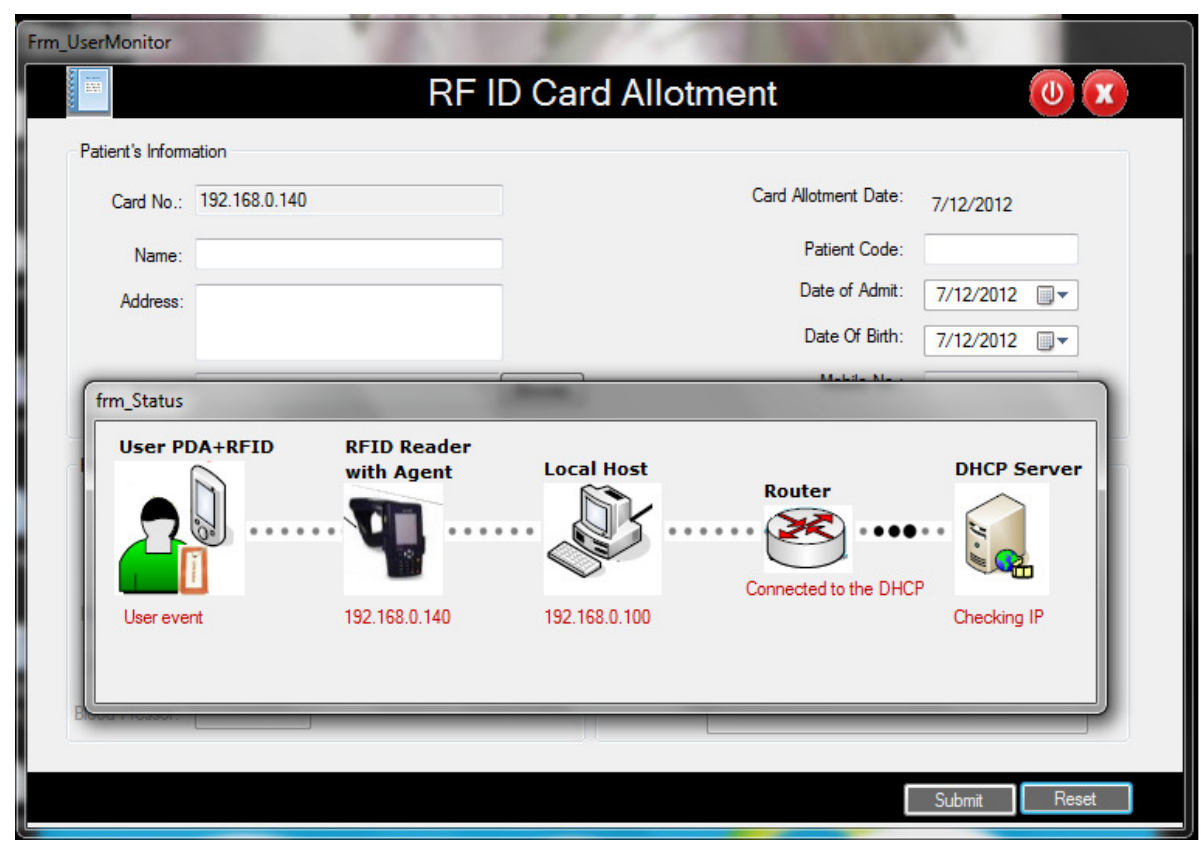

Figure 6: RFID card allotment and IP registration to ID server 
International Journal of Distributed and Parallel Systems (IJDPS) Vol.3, No.5, September 2012

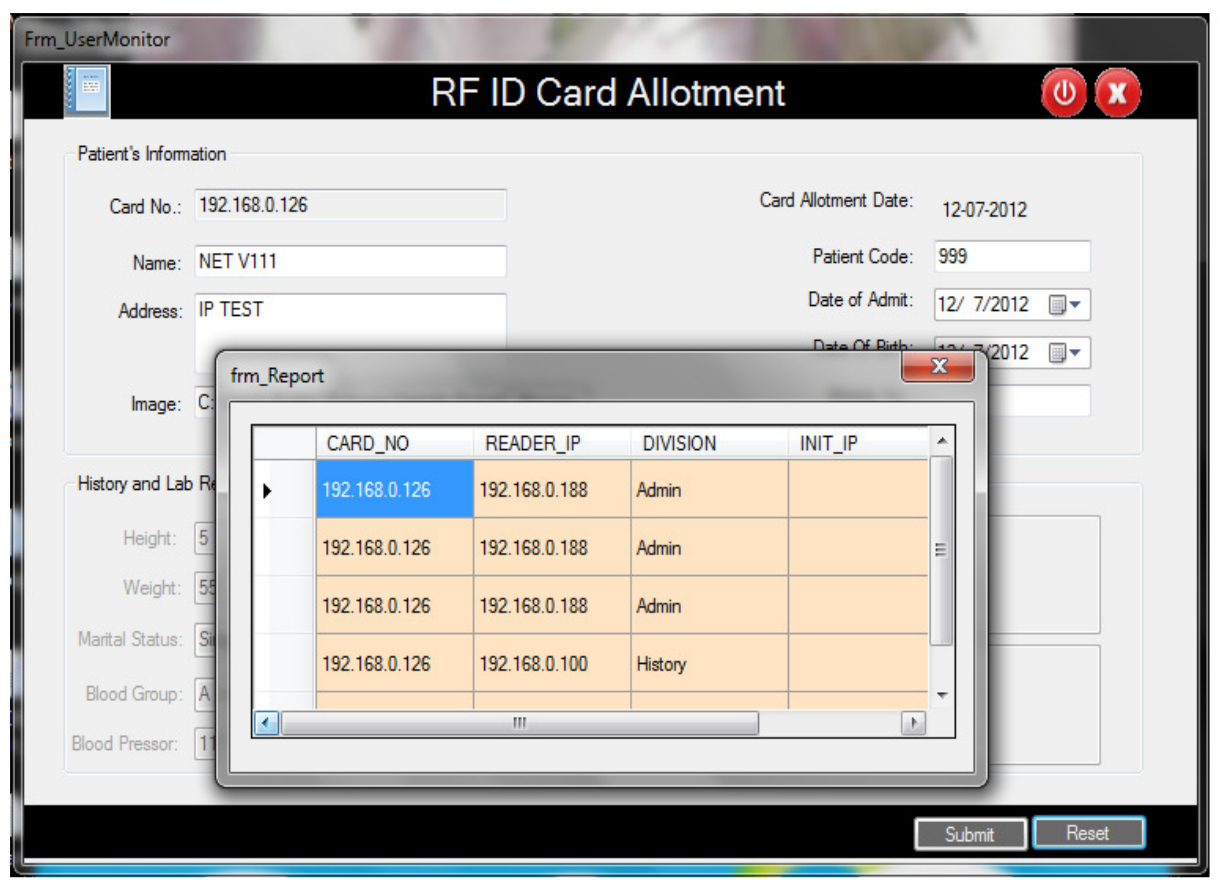

Figure 7: Status report of RFID tags

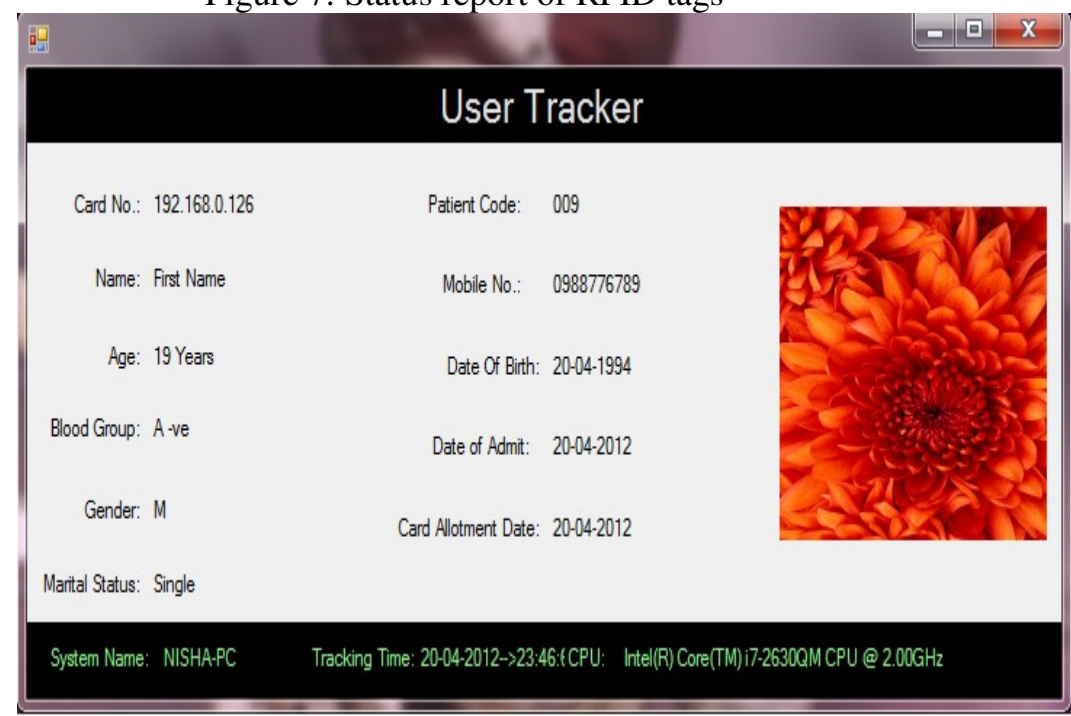

Figure 8: Tracking of objects using RFID reader

\section{CONCLUSION AND FUTURE WORK}

In this paper, integration of passive RFID technology in ubiquitous computing environment is proposed. RFID networking mechanism using RFID agent is used to establish networking using IP in RFID. The RFID agent can be positioned inside the RFID reader. Reader is given challenge to handle most of the complexity of the system. Mobility can be achieved using RFID tag and RFID agent as agent is the key element for RFID tag to IP address generation and management process. We can provide seamless mobility when any object moves to new network. Also using ID server any corresponding node in the network can communicate with RFID tag by using its mapped IP. 
Security is another important issue for a RFID-IP based ubiquitous networking. For securing IP communication, IPSec protocol can be used which provides authentication and confidentiality for IP communication. In the case of communication between $\mathrm{CN}$ and tagged objects, no additional security is required as they all are based on IP communication but if we allow direct communication from IP enabled RFID tag to $\mathrm{CN}$, it should be provided new layer of security.

Identification of object in ubiquitous networking environment by giving name to objects can be made possible in future by making use of Domain Name Server. By taking idea from [8] all the objects can be provided host identity by giving them name so that any client can search the location of object based on the name instead of using IP Address or UID of RFID tag. In future, IPV6 addressing can be used to identify all the objects as it gives more address space. IPV6 address can be generated from RFID tags in which subnet prefix of network is used to generate subnet identifier while RFID tag identifier can be used to generate unique interface identifier.

\section{REFERENCES}

[1] www2.docm.mmu.ac.uk/STAFF/M.Stanton/UbiqLab/index.html.

[2] Gyu Myoung Lee, Jun Kyun Choi, Noel Crespi, “Object identification for ubiquitous networking,” ICACT’2009, pp. 255-259, February 2009.

[3] EPCglobal, "The EPCglobal Architecture Framework Version 1.2," EPCglobal, USA, 2007.

[4] D. W. Engels. "Comparison of the Electronic Product Code Identification Scheme \& the Internet protocol Address Identification Scheme", June 2002.

[5] L. Sang-Do, S. Myung-Ki, and K. Hyoung-Jun. “EPC vs.IPv6 mapping mechanism.” In The 9th International Conference on Advanced Communication Technology, volume 2,pages 12431245, February 2007.

[6] RFC 2131 - dynamic Host Configuration Protocol.

[7] Dong Geun Yoon, Dong Hyeon Lee, Chang HoSeo, SeongGon Choi, "RFID Networking Mechanism Using Address Management Agent," $4^{\text {th }}$ Networked Computing and Advanced Information Management(NCM2008), vol. 1, pp. 617-622, Sept. 2008.

[8] Minami, M., Morikawa, H., \& Aoyama, T. 2004). "The design of naming-based service composition system for ubiquitous computing applications." Proceedings of the 2004 International Symposium on Applications and the Internet Workshops (pp. 304 - 312.) Los Alamitos: IEEE Computer Society.

\section{Authors}

Nisha J. Vaghela was born in India in 1984. She received B.E degree in Information Technology from saurashtra university in 2005. She is currently pursuing M.E in Computer Engineering from Pune University, India.

Parikshit N. Mahalle was born in India in 1979. He received his B.E. in Computer Engineering and M.E. in Computer Engineering from Pune university, India. He is currently pursuing his Phd from Aalborg university, Danmark. He is currently working as an assistant professor on SKN college of engineering, Pune. His area of research includes security in wireless communication.
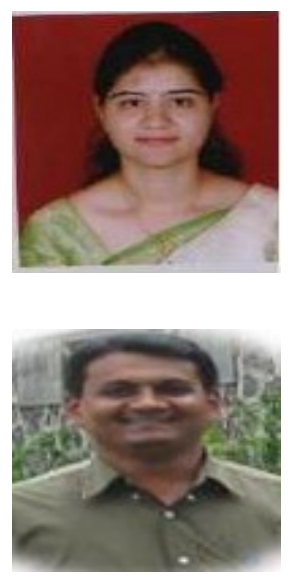